\title{
Disfunciones en el deseo sexual y su relación con los niveles de satisfacción en universitarios
}

\author{
Verónica Cervigón Carrasco \\ al287535@uji.es \\ Naiara Martínez Gómez \\ gomezn@uji.es \\ Yarisa Nicola \\ yarisa.nicola@gmail.com \\ María López-Fando Galdón \\ al320113@uji.es \\ Cristina Giménez García \\ gimenezc@uji.es
}

\section{Resumen}

Introducción: Las disfunciones en el deseo sexual, según la literatura, afectarían a cualquier edad y correlacionarían con menor satisfacción. Sin embargo, no siempre son atendidas en jóvenes. El objetivo del estudio es explorar su prevalencia en dicha población y su posible relación con la satisfacción de vida, de vida sexual y con su cuerpo y genitales. Se hipotetiza que las personas afectadas por el deseo tengan menor calidad de vida global y sexual, siendo más prevalente la interferencia del bajo deseo entre mujeres y del elevado deseo entre hombres.

Método: Para ello, 1841 personas $(61,2 \%$ mujeres) con edad promedio de 21,42 años cumplimentaron una encuesta sobre hábitos y experiencias sexuales.

Resultados: Se observa como un $30 \%$ de las personas presentan alteraciones asociadas a bajo deseo sexual, mientras un $40,6 \%$ las presenta respecto al deseo elevado. Según sexo, en mujeres es más prevalente el bajo deseo sexual $(34 \%)$ que en hombres $(23 \%)$ y más prevalente el excesivo deseo sexual en hombres (48\%) que en mujeres (36\%). La relación entre el deseo y la satisfacción a diferentes niveles arroja que, mientras el bajo deseo correlaciona negativa y significativamente $(p \leq 0,01)$ con todas las esferas, el excesivo deseo sexual lo hace con cuerpo $(p \leq 0,01)$ y vida global $(p \leq 0,05)$.

Discusión: Tal y como apunta la literatura, las mujeres presentan mayores niveles de bajo deseo y los hombres de alto, correlacionado con malestar vital y otros aspectos. Así pues, en el desarrollo de estrategias preventivas e intervención clínica, cabría tener en cuenta este hallazgo.

Palabras clave: disfunciones sexuales, bajo deseo sexual, excesivo deseo sexual, satisfacción, jóvenes. 


\section{Abstract}

Introduction: According to literature, sexual desire disorders would be related to low satisfaction at any age. Nevertheless, most of interventions have usually excluded young people. The main purpose of this study is to explore the prevalence of sexual desire disorders in this population, as well as their association with life and sexual satisfaction, and genital and body appearance satisfaction. We hypothesize that people with maladjusted desire will report lower life and sexual satisfaction; women will exceed men in problems with low desire meanwhile men will exceed women in problems with high desire.

Method: 1841 participants (61.2\% women), with average age of 21.42 years, completed a survey about sexual habits and experiences.

Results: $30 \%$ of participants have alterations related to low desire, while $40.6 \%$ of them reporte problems due to high sexual desire. According to sex, women showed lower sexual desire (34\%) compared to men $(23 \%)$, and men reported higher sexual desire $(48 \%)$ that women did $(36 \%)$. Regarding the relation between desire and satisfaction in different area, low sexual desire is associated with all areas negatively and significantly $(p \leqslant 0.01)$, and high sexual desire with body $(p \leqslant 0.01)$ and global life $(p \leqslant 0.05)$.

Discussion: According to literature, women show lower sexual desire and men higher sexual desire; both are related to life dissatisfaction and dissatisfaction in other aspects. Thus, preventive strategies and clinic intervention should consider this information.

Keywords: sexual dysfunctions, low sexual desire, high sexual desire, satisfaction, young people.

\section{Introducción}

La sexualidad se constituye como una dimensión inherente a la persona y, como tal, es una esfera significante e influyente en su vida y en su buen funcionamiento. Por ello, en los últimos años, ha aumentado la literatura científica relativa a su estudio y al de sus problemáticas asociadas (Cabello 2010; Shahmoradi y Saadat 2018). Dentro del espectro de la sexualidad, uno de los campos de mayor interés para su estudio es el área de las disfunciones sexuales (Ballester y Gil 1995). Por un lado, este interés nace de la elevada tasa de demanda de atención en los recursos sanitarios, indicador de una gran prevalencia de las disfunciones sexuales en la población. En un estudio realizado en Estados Unidos, el $43 \%$ de las mujeres y el $31 \%$ de los hombres presentaba alguna disfunción sexual (Martínez Ramírez, Sánchez Bravo, Carreño Meléndez y Gómez López 2005). Por otro lado, se ha visto que las disfunciones sexuales se asocian a un gran impacto en áreas como las relaciones interpersonales, la satisfacción vital o la calidad de vida de los afectados (Colson, Lemaire, Pinton, Hamidi y Klein 2006; Launmann, Paik y Rosen 1999).

Las disfunciones sexuales son un conjunto de síndromes que se caracterizan por alteraciones en el deseo sexual y una serie de cambios en las diferentes fases del ciclo de la respuesta sexual humana y que se dan en ambos sexos (Laumann, Paik y Rosen 1999). Estas se conceptualizaron, por tanto, a partir de la definición del ciclo de 
la respuesta sexual humana realizada por Masters y Johnson en el que estipulan 5 fases diferenciadas en dicho ciclo: deseo, excitación, meseta, orgasmo y resolución (Masters, Jonhson y Kolodny 1987). Debido a su presencia y elevada prevalencia en ambos sexos, las alteraciones en el deseo sexual destacan entre el resto de disfunciones sexuales, dándose tanto por bajo deseo o deseo hipoactivo como por deseo excesivo o elevado (Cabello 2010; Herrera 2003).

Por lo que respecta al bajo deseo sexual, se trata de una de las afecciones sexuales más prevalentes en la población, estimándose su prevalencia entre el $27 \%$ y el $32 \%$ (Shahmoradi y Saadat 2018). En esta línea, en el estudio de Martínez Ramírez, Sánchez Bravo, Carreño Meléndez y Gómez López (2005) se observa cómo el bajo deseo sexual ocupa el tercer puesto entre las disfunciones sexuales presentes tanto en hombres como en mujeres y el primer puesto en común en ambos. De hecho, Ballester y Gil (1995) encuentran una prevalencia del bajo deseo sexual del 37,2 \% en mujeres y del $24,6 \%$ en hombres; esta prevalencia es, por tanto, elevada en ambos sexos, pero se describen diferencias significativas a favor de las mujeres. Cuando se trata de analizar la prevalencia de esta disfunción atendiendo al momento evolutivo, lo que se encuentra es que su estudio se vincula de forma mayoritaria con la adultez tardía y la tercera edad (Herrera 2003), desatendiendo casi por completo a la población más joven. Sin embargo, es lógico pensar que, si la sexualidad se da a lo largo de todo el ciclo vital, las problemáticas vinculadas a esta también. Hace ya casi dos décadas se encontró en esta línea que en EE. UU. el $32 \%$ de las mujeres y el $13 \%$ de los hombres con edades comprendidas entre los 18 y 29 años presentaban alteraciones de bajo deseo sexual, evidenciando la presencia de bajo deseo en población joven (Launmann, Paik y Rosen 1999).

En el opuesto nos encontramos con el deseo sexual elevado, el cual, aunque no constituye una disfunción sexual per se, forma parte de otras problemáticas más extensas y complejas como el trastorno por hipersexualidad (American Psychiatric Association 2013) o la adicción al sexo (Echeburúa 2012). La hipersexualidad es un cuadro clínico que se caracteriza, entre otros aspectos, por un excesivo deseo sexual que se escapa del control del sujeto. Este trastorno se estima presente en un $6 \%$ de la población, encontrándose su prevalencia en un 3-17,4\% en hombres y un 1,2$32,2 \%$ en mujeres (Castro, Ballester y Gil 2017). A pesar de la evidencia de que el deseo sexual elevado es determinante en esta patología, no existen estudios reconocidos que determinen su prevalencia, siendo esta seguramente más alta que la propia del trastorno de hipersexualidad, puesto que los niveles de deseo sexual elevados no siempre van aparejados de adicción o hipersexualidad (Echeburúa 2012). Su estudio en población joven sería conveniente, puesto que durante este momento vital es cuando se empiezan a desarrollar las problemáticas vinculadas a la sexualidad excesiva e incontrolada, es decir, un patrón sexual patológico (Castro, Ballester y Gil 2017).

Como se ha adelantado previamente, gran parte de la gravedad de las disfunciones sexuales se encuentra en el impacto que pueden llegar a tener sobre los afectados. Gran número de estudios vinculan la experiencia de las disfunciones sexuales con una menor calidad de vida (Colson, Lemaire, Pinton, Hamidi y Klein 2006; Launmann et al. 2005; Martínez Ramírez, Sánchez Bravo, Carreño Meléndez y Gómez López 2005; Shahmoradi y Saadat 2018), peor salud mental, estrés, peor calidad de relaciones interpersonales y menor felicidad (Colson, Lemaire, Pinton, Hamidi y Kelin 2006; Launmann, Paik y Rosen 2005). Además, se ha visto que correlacionarían también con gran número de experiencias y relaciones insatisfactorias tanto emocionales como físicas, es decir, con menores niveles de satisfacción en diferentes esferas. Esta correlación se encontraría asociada a todas las disfunciones, pero sería especialmente 
fuerte y significativa con deseo sexual bajo $y$, de forma más severa, en mujeres (Launman et al. 2005).

Por tanto, los dos principales objetivos de este estudio son, por un lado, explorar la prevalencia de problemáticas vinculadas al deseo sexual en población joven y, por otro lado, analizar la posible relación existente con los niveles de satisfacción en diferentes áreas: vida global, vida sexual, cuerpo y genitales. A la luz de los datos presentados, se hipotetiza sobre la existencia de una mayor interferencia del bajo deseo sexual en mujeres y del elevado deseo sexual en hombres. También se hipotetiza que las personas con afectación en el deseo sexual, independientemente de la dirección de esta, presenten menor calidad de vida general y sexual.

\section{Método}

\section{Participantes}

En total participaron 1841 personas, con edades que oscilan entre los 18 y 83 años, siendo la media de edad de 21,42 años y la desviación típica de 5,53 . Por lo que respecta a la distribución por sexos, encontramos que el $61,2 \%$ de la muestra se componía de mujeres y el $38,8 \%$ restante eran hombres.

En cuanto al nivel de estudios alcanzado, la mayoría tenían estudios universitarios $(64,8 \%$ grado y $4,9 \%$ posgrado) y el resto tenían estudios secundarios o de formación profesional $(28,6 \%)$. Respecto a la orientación sexual, el $88,1 \%$ de la muestra era heterosexual, el $7,6 \%$ era bisexual, el $3,3 \%$ era homosexual y el $0,4 \%$ se consideraba asexual.

\section{Instrumento}

En el presente estudio se ha usado el Cuestionario de Hábitos y Experiencias Sexuales realizado por Salusex en el 2017, en el que se evalúan diferentes as pectos y esferas vinculadas a la sexualidad. Para este estudio se han usado tres áreas concretas del instrumento:

- Datos sociodemográficos con el fin de obtener información relativa a las características de la muestra obtenida: sexo, edad, orientación, nivel de estudios.

- Subescala relativa a disfunciones sexuales: en este caso se han utilizado dos ítems dicotómicos presentes en la subescala: presencia o ausencia de bajo deseo sexual y presencia o ausencia de elevado deseo sexual.

- Subescala relativa a la satisfacción: dicha subescala se compone de 5 ítems, de los cuales se han seleccionado 4 , que son los relativos al grado de satisfacción con la vida general, la vida sexual, el cuerpo y los genitales, valorados en una escala Likert como $0=$ Nada satisfecho y $3=$ Muy satisfecho.

\section{Procedimiento}

La información del estudio se divulgó de forma presencial a través de actividades de educación para la salud, desarrolladas en el campus de la Universitat Jaume I y dirigidas a prevenir las conductas sexuales de riesgo. Se informaba a las personas sobre el objetivo y se les pedía que cumplimentaran el cuestionario sobre hábitos y experiencias sexuales. Una vez daban su consentimiento informado de forma oral tras 
ser informados sobre el contenido del cuestionario y su finalidad, cumplimentaban el instrumento en formato papel y bolígrafo, en aproximadamente unos 20 minutos. La participación era voluntaria, anónima e individual en diferentes espacios habilitados para preservar su intimidad. Mediante este procedimiento se logró una participación de 1931 personas $(100 \%)$, de las que, finalmente, fueron incluidas en el estudio 1841 personas $(95,3 \%)$. Los 90 participantes restantes $(4,7 \%)$ fueron excluidos por no cumplimentar el instrumento correctamente o en su totalidad.

\section{Resultados}

Con el objetivo de explorar la prevalencia de las disfunciones en el deseo sexual en la población universitaria, se analizaron los ítems dicotómicos «¿Alguna vez has mostrado problemas de bajo deseo sexual?» y "¿Alguna vez has mostrado problemas de excesivo deseo sexual?». Lo que se obtuvo fue que ambas experiencias son altamente prevalentes en población universitaria, puesto que un $28,6 \%$ de la población evaluada manifestaba presentar problemas vinculados al bajo deseo sexual, mientras que el $38,7 \%$ los reportaba vinculados al excesivo deseo sexual. En función del sexo, se observa cómo es una variable diferencial en cuanto a la dirección de la alteración en el deseo. Se realiza una prueba t para muestras independientes y lo que se obtiene es que, por un lado, el bajo deseo sexual es más prevalente en mujeres (34 \%) que en hombres (23\%) ( $t=5,323)$, mientras que, por otro lado, el elevado deseo sexual se encuentra en mayor proporción en hombres (48\%) que en mujeres (36\%), siendo por tanto más prevalente en los primeros $(t=5,385)$. Cuando se compara la prevalencia de ambas disfunciones en ambos sexos, lo que se encuentra es que el elevado deseo sexual es más prevalente que el bajo deseo sexual en la muestra total (véase la tabla 1).

Tabla 1

Distribución de las alteraciones en el deseo sexual en general y por sexos (\%)

Ítems Hotal Hombres Mujeres

¿Alguna vez has mostrado problemas de $\quad 28,6 \% \quad 34 \%$ bajo deseo sexual?

¿Alguna vez has mostrado problemas de $\quad 38,7 \% \quad 48 \% \quad 36 \%$ excesivo deseo sexual?

Para analizar la relación existente entre las alteraciones en el deseo sexual y la satisfacción de los participantes en las diferentes esferas se calculó el coeficiente de correlación de Pearson para ambas disfunciones en cada esfera. Lo que se obtuvo respecto al bajo deseo sexual es que este correlaciona negativa y significativamente con todas las esferas de satisfacción evaluadas a un nivel de significación $p<0,01$. La mayor correlación del bajo deseo sexual se da con la satisfacción con el cuerpo, seguida de la falta de satisfacción con los genitales, con la vida en general y, por último, con la vida sexual. En cuanto al excesivo deseo sexual, solo se encuentra que correlaciona significativamente y de forma negativa con la satisfacción con el cuerpo y con la satisfacción con la vida en general (véase la tabla 2). 
Tabla 2

Correlaciones entre alteraciones en el deseo sexual y niveles de satisfacción en diferentes esferas

$\begin{array}{lcc} & \text { Bajo deseo sexual } & \text { Elevado deseo sexual } \\ \text { Vida general } & -\mathbf{0 , 1 2 7 ^ { * * }} & \mathbf{- 0 , 0 5 6 ^ { * }} \\ \text { Vida sexual } & -\mathbf{0 , 0 8 7 ^ { * * }} & -0,012 \\ \text { Cuerpo } & -\mathbf{0 , 1 4 8 ^ { * * }} & \mathbf{- 0 , 0 6 1 ^ { * * }} \\ \text { Genitales } & \mathbf{- 0 , 1 3 5 ^ { * * }} & 0,030 \\ \text { Niveles de significación: } p<0,05^{*}, p<0,01^{* *} & \end{array}$

\section{Discusión}

Uno de los objetivos de nuestro estudio era explorar la prevalencia de las alteraciones en el deseo sexual en población joven, desatendida, casi por completo, en el campo del estudio de las disfunciones sexuales. Lo que se ha encontrado es que, tal y como apunta la literatura revisada (Cabello 2010; Launmann, Paik y Rosen 1999), las disfunciones sexuales en general, y las alteraciones en el deseo sexual en concreto, al igual que en otros colectivos, se dan también en población joven. Esto encuentra su motivo en que la sexualidad es una dimensión inherente a la persona y, como tal, la acompaña a lo largo de la vida, de manera que las variables relacionadas con esta, entre las que se encuentran las disfunciones sexuales, son susceptibles de presentarse en cualquier momento evolutivo, adolescencia y juventud incluidas.

Se ha encontrado que, como se había hipotetizado al inicio del estudio, el sexo es una variable que influye en la dirección e interferencia de las alteraciones en el deseo sexual. Los datos muestran una mayor prevalencia y, por tanto, mayor interferencia del bajo deseo sexual en las mujeres y del elevado deseo sexual en los hombres. Los resultados obtenidos por lo que respecta a la mayor prevalencia del bajo deseo sexual en mujeres van en la línea de la literatura revisada anteriormente, donde también se describen mayores porcentajes de dicha disfunción en el colectivo femenino (Ballester y Gil 1995; Launmann, Paik y Rosen 1999). Entre las posibles causas que darían origen a este efecto diferencial en la interferencia podríamos apuntar a que, históricamente, las mujeres han tenido un desarrollo de la sexualidad mucho más restringido y limitado que los hombres y al substrato cultural en el que nos hemos movido, en el que la concepción de la mujer como ser sexual se rechazaba en gran parte de los círculos sociales (Herrera 2003). En cuanto a los resultados obtenidos en relación con el deseo sexual elevado, se ha visto que, efectivamente, y tal y como se había hipotetizado, la interferencia es mayor en los hombres. Una de las posibles explicaciones de este fenómeno podría residir en la dada con anterioridad para explicar la interferencia del bajo deseo sexual en mujeres, pero, en este caso, a la inversa. Es decir, los hombres han recibido en general una educación más liberal en cuanto a sexualidad que las mujeres y no se les ha limitado tanto desde sus círculos sociales, puesto que su sexualidad era asumida como normal. Además, estos resultados irían en sintonía con el hecho de que en la literatura científica se postule al hombre como principal afectado por la adicción al sexo o la hipersexualidad, trastorno en el que ya se ha comentado que el elevado deseo sexual es central y determinante (Castro, Ballester y Gil 2017; Echeburúa 2012).

El otro objetivo de este estudio era explorar la posible relación existente entre ambas alteraciones en el deseo sexual y los niveles de satisfacción en diferentes esferas: vida 
global, vida sexual, cuerpo y genitales. Como hipótesis de partida, en relación con este objetivo, se había postulado que aquellas personas afectadas por la presencia de problemas en el deseo sexual presentarían menores niveles de satisfacción y, por tanto, menor calidad de vida. A la luz de los datos obtenidos se puede afirmar que dicha hipótesis se cumple, puesto que ambas alteraciones muestran correlaciones negativas con áreas de satisfacción. Estos mismos datos muestran que, mientras que el elevado deseo sexual se relaciona significativamente y de forma negativa con satisfacción con la vida y con el cuerpo, el bajo deseo sexual lo hace de forma significativa y negativa con todas las áreas evaluadas, lo que se puede interpretar como que el bajo deseo sexual genera un mayor menoscabo e impacto, siendo por tanto de mayor gravedad para el afectado. Este hallazgo no es nuevo, pero reafirma lo ya visto, puesto que es congruente con lo encontrado en otros estudios como el de Launmann, Paik y Rosen (1999), donde se postula también que el impacto es mayor y más negativo en las mujeres, lo cual es lógico si atendemos a que en el bajo deseo sexual reside mayor gravedad y este se erige más prevalente en mujeres.

Como una de las posibles limitaciones de este estudio se encuentra el tamaño de la muestra, la cual es pequeña teniendo en cuenta el objetivo del estudio, por lo que en estudios futuros relacionados con la temática abordada sería conveniente ampliarla con el fin de que los datos obtenidos sean más representativos y cercanos a la realidad estudiada. Además de ampliarla dentro de la misma universidad, sería conveniente ampliar el estudio a población universitaria de otros emplazamientos con la finalidad de lograr la máxima representatividad de los datos obtenidos. Por otro lado, utilizar otros autoinformes destinados en específico a los constructos evaluados hubiera otorgado a la investigación un mayor valor ecológico, puesto que el instrumento utilizado realiza un barrido por diferentes variables vinculadas a los hábitos y las experiencias sexuales, pero lo hace sin focalizarse en ninguna en especial. Su uso en futuras investigaciones relativas a este tema sería recomendable.

A partir de estos datos y de lo expuesto, queda clara la importancia de desarrollar acciones y estrategias preventivas destinadas al abordaje de las disfunciones sexuales, en este caso, de las alteraciones en el deseo sexual, que incluyan a la población joven en la población diana, la que se ha demostrado también afectada y en gran proporción por estas. Además, en dichas acciones resultaría fundamental incluir componentes, actividades o módulos temáticos en los que se trabaje y se aborde de forma específica y exhaustiva la satisfacción como constructo, tanto desde el punto de vista teórico como práctico. Ello sería con el objetivo de reducir el impacto negativo de las problemáticas expuestas, puesto que los niveles de satisfacción se han visto fuertemente asociados con aspectos como la calidad de vida y el bienestar a diferentes niveles.

\section{Referencias bibliográficas}

American Psychiatric Association. 2013. Diagnostic and statistical manual of mental disorders. 5th Ed. Washington, DC: American Psychiatric Association.

Ballester, Rafael y Maria Dolores Gil. 1995. «Estudio epidemiológico sobre la prevalencia de disfunciones sexuales en la Comunidad Valenciana». Psicothema, 7(1): 95-104

Cabello, Francisco. 2010. Manual de sexología y terapia sexual. Madrid: Síntesis.

Castro, Jesús, Rafael Ballester y Maria Dolores Gil. 2017. «Validación preliminar del inventario de hipersexualidad en jóvenes». Àgora de Salut, 4(6): 53-64

Colson, Marie Helene, Antoine Lemaire, Philippe Pinton, Karim Hamidi y Patrick Klein. 2006. «Original Research - Couples' Sexual Dysfunction: Sexual Behaviors 
and Mental Perception, Satisfaction and Expectations of Sex Life in Men and Women in France». The Journal of Sexual Medicine, 3(1): 121-131

Echeburúa, Enrique. 2012. "¿Existe realmente la adicción al sexo?». Adicciones, 24(6): 281-286.

Herrera, Adela. 2003. «Sexualidad en la vejez: ¿Mito o realidad?». Revista chilena de obstetricia y ginecología, 68(2): 150-162.

Launmann, Edward, Anthony Paik y Raimond Rosen. 1999. «Sexual dysfunction in the United States». Journal of American Medical Association, 281(6), 537-554.

Launmann, Edward, Alfredo Nicolosi, Dale B. Glasser, Anthony Paik, Clive Gingell, Edson Moreira y Tianfu Wang. 2005. "Sexual problems among women and men afed 40-80 years: prevalence and correlates identified in the Global Study of Sexual Attitudes and Behaviors». International Journal of Impotence Research, 17, 39-57.

Martínez Ramírez, Susana, Claudia Sánchez Bravo, Jorge Carreño Meléndez y María Eugenia Gómez López. 2005. «Disfunciones sexuales femeninas y masculinas: comparación de género en una muestra de México». Salud Mental, 28(4): 7480.

Masters, H. William, Virginia E. Johnson y Robert C. Kolodny. 1987. La sexualidad humana: perspectivas clínicas y sociales. Barcelona: Grabaljo.

Shahmoradi, Somayeh y Seyed Hassan Saadat. 2018. «Epidemiology and Associated Risk Factors for Sexual Dysfunction: A systematic Review». Journal of Research in Medical and Dental Sicence, 6(4): 69-73. 